\title{
Topological symmetry breaking in viscous coarsening
}

\author{
David Bouttes, ${ }^{1}$ Emmanuelle Gouillart, ${ }^{2}$ and Damien Vandembroucq ${ }^{1}$ \\ ${ }^{1}$ Laboratoire PMMH, UMR 7636 CNRS/ESPCI/Univ. Paris 6 UPMC/Univ. Paris 7 Diderot, \\ 10 rue Vauquelin, 75231 Paris cedex 05, France \\ ${ }^{2}$ Surface du Verre et Interfaces, UMR 125 CNRS/Saint-Gobain, 93303 Aubervilliers, France
}

(Dated: June 17, 2022)

\begin{abstract}
The crucial role of hydrodynamic pinch-off instabilities is evidenced in the coarsening stage of viscous liquids. The phase separation of a barium borosilicate glass melt is studied by in-situ synchrotron X-Ray tomography at high temperature. The high viscosity contrast between the less viscous phase and the more viscous phase induces a topological symmetry breaking: capillary breakups occur preferentially in the less viscous phase. As a result, contrasting morphologies are obtained in the two phases. This symmetry breaking is illustrated on three different glass compositions, corresponding to different volume fractions of the two phases. In particular, a fragmentation phenomenon, reminiscent of the end-pinching mechanism proposed by Stone et al. 1, 2, is evidenced in the less viscous phase.
\end{abstract}

Understanding the fragmentation of liquids is a longstanding problem in fluid dynamics $[3]$. The competition between surface tension, inertial and viscous effects at play in droplet generation and spray formation is of paramount importance in fields such as microfluidics [4], ocean-atmosphere exchanges [5] or volcanic eruptions [6, 7]. Of particular interest for industrial applications is the size distribution arising from the fragmentation process [3].

In the context of viscous coarsening of phase separated silicate melts, we have recently evidenced an original fragmentation phenomenon leading to a power-law size distribution of droplets 8, 9. Here the scale-free character of the fragment-size distribution is a direct inheritance of the self-similar structure of the inter-connected cluster. In the course of liquid-liquid phase separation, interface tension driven coarsening induces a temporal growth of the characteristic size of the phase domains $\ell(t) \propto t^{\alpha}$ where the exponent $\alpha$ depends on the transport mechanism at play (diffusion, advection...) [10. A major feature of the coarsening phenomenology is thus the observation of self-similar structures obeying dynamical scaling invariance: structures are left statistically invariant after renormalization by the characteristic length scale $\ell(t)$.

In these experiments, as in other works, dynamical scaling is a powerful tool to describe the statistical features of the coarsening [11, 12, and can be derived in a few situations [13. The starting point of the scaling laws governing domain growth is often the understanding of the local mechanisms at play, e.g. hydrodynamic pinch-off for viscous coarsening. The importance of these local mechanisms was stressed by Siggia in his pioneering work [14, but they have received little attention [15-17], despite being key to the specific geometrical features of the coarsening, such as the fragmentation we observed.

Here we show that a viscosity contrast can break the symmetry between the two phases and lead to a different morphology of the two phases, and to the fragmentation of the less viscous phase. We use in situ synchrotron microtomography to follow the temporal development of viscous coarsening in phase-separated barium borosilicate melts at high temperature, with sufficient spatial and temporal resolution to access the fine details of the evolution of the structure of the two liquids. In particular, we unveil the crucial importance of the hydrodynamic pinch-off mechanisms at play in viscous coarsening and the effect of a viscosity contrast.

Phase-separated barium-borosilicate glasses - In the following we present results obtained on three glasses hereafter denoted by $\mathrm{G}_{1}, \mathrm{G}_{2}, \mathrm{G}_{3}$ (see compositions in Table I). These glasses lie on the same tie-line: when heated in the range $T=1160^{\circ} \mathrm{C}-1210^{\circ} \mathrm{C}$ used in the present series of experiments, they decompose into a viscous silica-rich phase and a fluid barium-rich phase. The viscosity contrast between the two phases is about 5 orders of magnitudes 9. Within the limits of the precision of the formulation and the control and variarions of temperatures, the composition of the two separated phases obtained in the different experiments are extremely similar [18. As summarized in Table I, only the volume fraction of the two phases depends on the initial composition. Here the volume fractions of the fluid phase are respectively $\Phi_{1}=0.27, \Phi_{2}=0.45$ and $\Phi_{3}=0.72$. For the three volume fractions, the initial microstructure is bicontinuous, suggesting spinodal decomposition.

In-situ experiments - X-ray tomographic experiments have been performed on beamline ID19 at the European Synchrotron Radiation Facility (ESRF). Glass samples $2 \mathrm{~mm}$ in diameter were studied in situ at high temperatures using a dedicated furnace. A high-flux pink beam of energy $32 \mathrm{keV}$ was used, so that it took $15 \mathrm{~s}$ to acquire a full 3-D image, corresponding to a volume of size $700 \mu \mathrm{m} \times 700 \mu \mathrm{m} \times 350 \mu \mathrm{m}$, with voxels of size $1.1 \mu \mathrm{m}$. Additional details on the experimental set-up and data processing (reconstruction, segmentation and characterization of the 3D geometry) can be found in Ref. [8, 9, 18].

Coarsening results from series of topological events Coarsening studies emphasize the scaling properties of 


\begin{tabular}{c|c|c|c|c|c}
$\begin{array}{c}\text { Glass } \\
\text { compositions }\end{array}$ & $\mathrm{SiO}_{2}$ & $\mathrm{~B}_{2} \mathrm{O}_{3}$ & $\mathrm{BaO}$ & $\mathrm{T}\left({ }^{\circ} \mathrm{C}\right)$ & $\begin{array}{c}\text { Fluid phase } \\
\text { vol. } \%\end{array}$ \\
\hline $\mathrm{G}_{1}$ & 65 & 17 & 18 & 1160 & $27 \pm 2$ \\
$\mathrm{G}_{2}$ & 60 & 19 & 21 & 1210 & $45 \pm 2$ \\
$\mathrm{G}_{3}$ & 50 & 22 & 28 & 1160 & $72 \pm 2$ \\
Viscous phase & 80 & 17 & 3 & & 0 \\
Fluid phase & $36-38$ & 26 & $36-38$ & & 100
\end{tabular}

TABLE I. Compositions in weight percentages of the three glass compositions $\mathrm{G}_{1}, \mathrm{G}_{2}, \mathrm{G}_{3}$ under study and of the two phases, (viscous) silica-rich and (fluid) barium-rich respectively, obtained after separation. The temperatures of heat treatments are also indicated.
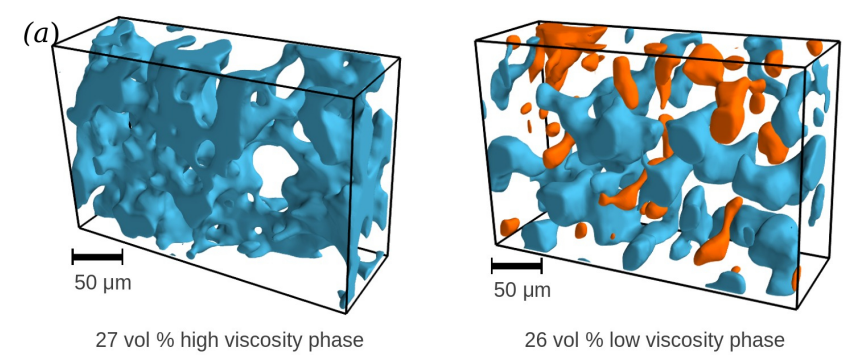

26 vol \% low viscosity phase

FIG. 1. Evolution of the topology of the minority (bariumrich) phase of glass $\mathrm{G}_{2}$ under coarsening. Top: snapshots of an elementary topological event, the rupture of a capillary bridge. Bottom: illustration of the same event in the complementary (silica-rich) phase, now the resorption of a hole.

domain growth and usually ignore the details of the geometrical changes at play at the local scale. Independently of the nature of the transport mechanism (diffusion or advection), statistical invariance upon time has important consequences from a topological point of view. The characteristic length scale of the structure can be interpreted as a typical mesh size and the growth of this length scale implies a decrease of the number of loops of the structure, hence a decrease of the topological genus of the structure. However it is not possible to change the genus of a structure by a continuous deformation. Coarsening results necessarily from a sequence of topological events: ruptures of links and their complementaries, resorptions of loops. This point has been recognized early on by Siggia [14, who proposed that viscous coarsening results from capillary breakups and retractions of ligaments.

As illustrated on Fig.1(top) obtained on glass $\mathrm{G}_{2}$, such pinch-off events can be identified along the coarsening stage. A capillary bridge breaks up and the retraction of the resulting ligament feeds the remaining structurehence the growth of its characteristic size. As shown in Fig. 1 (bottom), the same elementary event can be seen as the retraction of a loop in the complementary phase.

Beyond this example of topological event at local scale, the Gauss-Bonnet theorem gives a global mathematical characterization of the deep link between domain growth

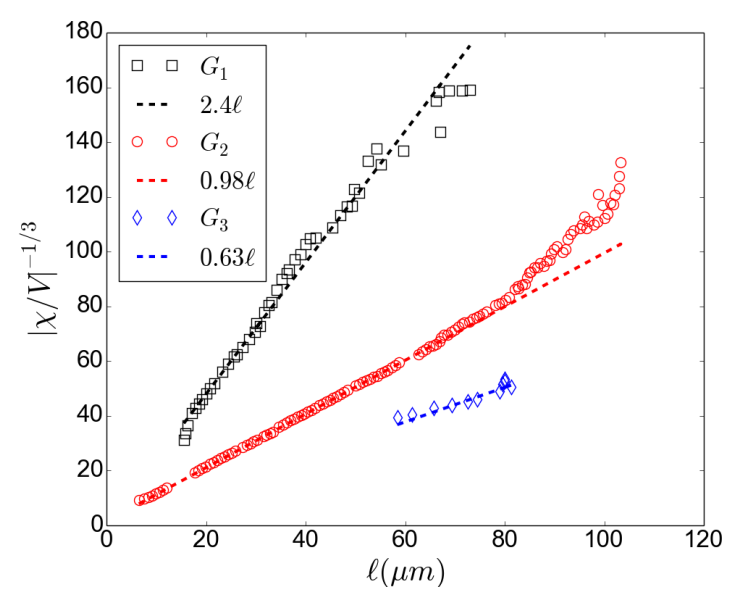

FIG. 2. Evolution under coarsening of the inverse cubic root of the volumic Euler characteristic $\chi$ of the percolating domain vs. characteristic size $\ell$ for the three glasses $G_{1}, G_{2}$ and $\mathrm{G}_{3}$. The dotted lines show indicative linear behaviors associated with the expected scaling for viscous coarsening.

and topology [19, 20]:

$$
\int_{S} K \mathrm{~d} S=2 \pi \chi
$$

The integral of the Gaussian curvature $K$ on the surface of the domains gives immediately access to the Euler characteristic $\chi$, a topological invariant which is the sum of isolated objects $\mathcal{N}$, minus the number of loops $\mathcal{L}$, plus the number of cavities $\mathcal{O}: \chi=\mathcal{N}-\mathcal{L}+\mathcal{O}$. During coarsening, we expect capillary bridges to break, hence decreasing the number of loops and the absolute value of the Euler characteristic. The dynamic scaling invariance at play for the evolution of the morphology of the domains thus directly translates to their topology 21, 22. Let us call $\ell(t)$ the characteristic length scale at time $t$. Dynamic invariance imposes that the statistical distribution of Gaussian curvatures $P[K(t)]$ obeys:

$$
P[K(t)]=\ell(t)^{2} \Psi\left[K(t) \ell(t)^{2}\right] .
$$

Combining (1) and (2) results in the following evolution of the volumetric Euler characteristic $\chi_{V}$ :

$$
\chi_{V}(t)=\frac{\chi(t)}{V} \simeq \frac{S}{V} \bar{K}(t) \simeq \ell(t)^{-3} \simeq\left(\frac{\gamma}{\eta} t\right)^{-3}
$$

where $S$ and $V$ are the total surface and volume of the domain. The interface tension $\gamma$ and the viscosity $\eta$ are associated with Siggia's scaling of viscous coarsening $\ell(t) \simeq(\gamma / \eta) t$, 9, 14].

Using the algorithm of Ref. [23 25] we computed the evolution under coarsening of $\chi_{V}$ versus the expected characteristic length scale $\ell(t)=V / S$, here computed on the largest (interconnected) domain of the fluid phase 
for the three liquids. In Fig. 2 we represent the evolution of the effective length scale $\ell_{\chi}(t)=\chi_{V}^{-1 / 3}(t)$ versus the coarsening characteristic length $\ell(t)$. The remarkable linear correlation that we obtain between the two quantities illustrates that dynamic scaling is valid for topological quantities (such as $\chi_{V}$ ) as well, and emphasizes the importance of a topological approach of coarsening [22].

Viscosity contrast induces topological symmetry breaking. The phase-separating melt under study is characterized by a strong viscosity contrast. In the range of temperatures considered $\left[1160^{\circ} \mathrm{C}-1210^{\circ} \mathrm{C}\right]$, the viscosities are $\eta_{F} \approx 10 \mathrm{~Pa}$.s for the fluid phase and $\eta_{V} \approx 10^{6} \mathrm{~Pa}$.s for the viscous phase [9, 18].

As discussed above, coarsening relies on a succession of capillary break-ups and retractions of ligaments. How is the dynamics affected by the strong viscosity asymmetry? From the early work of Tomotika [26] based on linear stability analysis, to the more recent discussions about end-pinching 1, 2, 27- 30 and detailed studies of the break-up [31 36], the question of the stability of a viscous thread or droplet suspended in another viscous fluid has raised a wide interest in fluid mechanics (see e.g. ref. 37 for a review). The present situation is clearly more difficult to handle for at least two reasons. First, the geometry of the inter-connected cluster and its ligaments is far more complex than a simple droplet or a thread. In particular different curvatures have to be considered. A closer configuration in this respect may be that of a toroidal droplet [38, 39]. Second, the liquid surrounding the ligament about to break up is not homogeneous but is itself a mixture of the two phases. An effective viscosity of the viscous matrix can be obtained using a homogenization approach. However, long-range hydrodynamic interactions are likely to be at play and introduce correlation between successive breaking events.

Forgetting about most of the complexity of the problem, we draw simple scaling arguments, and estimate the time scales associated with the two mechanisms at play: break-up and retraction of a ligament. The only length scale to be considered here is that of the coarsening $\ell(t)$.

Retraction - A typical estimate for the retraction time $\tau_{R}$ of a ligament of size $\ell$ and viscosity $\eta_{T}$ suspended in a fluid of viscosity $\eta_{M}$ is [40, 41]:

$$
\tau_{R} \approx \frac{3 \ell}{4} \frac{\eta_{T}+\eta_{M}}{\gamma}
$$

For a disordered matrix surrounding the ligament, a simple estimate of the effective viscosity gives $\eta_{M} \approx$ $\phi \eta_{F}+(1-\phi) \eta_{V} \approx(1-\phi) \eta_{V}$ [42. We thus obtain the retraction times of a fluid and a viscous ligament respectively:

$$
\tau_{R}^{F} \approx \frac{3}{4}(1-\phi) \frac{\eta_{V} \ell}{\gamma} \quad, \quad \tau_{R}^{V} \approx \frac{3}{4}(2-\phi) \frac{\eta_{V} \ell}{\gamma} .
$$

Despite the strong viscosity contrast the time scale is thus almost the same for the retraction of a fluid or a viscous ligament in a phase-separated melt.

Break-up - Generalizing the time scale for the final break-up of a ligament 43, 44, to the case of viscosity contrast 31, 35, we get

$$
\tau_{R} \approx H_{0}^{E} \frac{\eta_{M} \ell}{\gamma}\left(\frac{\eta_{T}}{\eta_{M}}\right)^{\alpha},
$$

where again $\eta_{M}$ is the viscosity of the suspending fluid and $\eta_{T}$ that of the ligament, the constant $H_{0}^{E} \approx 0.03[43$, 44 and the exponent $\alpha \approx 0.5-0.6$ [31, 35. For simplicity, in the following we consider $\alpha=0.5$ and get respectively for the break-up time of a fluid and a viscous ligament surrounded by the phase-separated mixture:

$$
\tau_{B}^{F} \approx \frac{\sqrt{1-\phi}}{H_{0}^{E}} \frac{\sqrt{\eta_{V} \eta_{F}} \ell}{\gamma}, \quad \tau_{B}^{V} \approx \frac{\sqrt{1-\phi}}{H_{0}^{E}} \frac{\eta_{V} \ell}{\gamma} .
$$

Unlike for retraction, the break-up time scale appears to be much lower for a fluid ligament than for its viscous counterpart. A first consequence is that ruptures of capillary bridges, as shown in Fig. 11 are much more likely in the fluid phase than in the viscous phase. Conversely, hole resorptions are more likely in the viscous phase. A second consequence is that the breaking time scale of a fluid ligament may also become much lower than the retraction time. This leaves room for an end-pinching like mechanism in the fluid phase, i.e. an additional rupture event during the retraction of a broken capillary bridge, leading to fragmentation. In contrast, we expect fragmentation to be very unlikely in the viscous phase.

Following these simple scaling arguments, viscosity contrast should induce a topological symmetry breaking during viscous coarsening. This is indeed the case. In the supplementary material (available on line) we show a movie corresponding to the series of 3D tomography images of the coarsening of glass $\mathrm{G}_{2}$. Only the minority fluid phase experiences fragmentation.

Another spectacular illustration of this topological symmetry breaking is given in Fig. 3(a) that shows the contrasting morphology of glasses $\mathrm{G}_{1}$ and $\mathrm{G}_{3}$ after 20 min of heat treatment. The two compositions "mirror" each other: in $\mathrm{G}_{1}$ the volume fraction of the fluid phase is $\Phi_{1}=0.27 \pm 0.02$ while it is $\Phi_{3}=0.72 \pm 0.02 \approx 1-\Phi_{1}$ in $G_{3}$. Here only the minority phases are represented, respectively the fluid phase for $\mathrm{G}_{1}$ and the viscous phase for $\mathrm{G}_{3}$. When the minority phase is the fluid one, a significant fragmentation is observed. Many isolated domains (here colored in orange) can be identified together with the percolating domain (in blue). In the complementary case, no fragmentation is observed in the minority viscous phase [45, only a fully connected domain is obtained. The shape of the interconnected cluster also differs between $G_{1}$ and $G_{3}$ : when composed of the fluid phase, it has a more compact and globular shape, while elongated structures are observed for the viscous phase in 


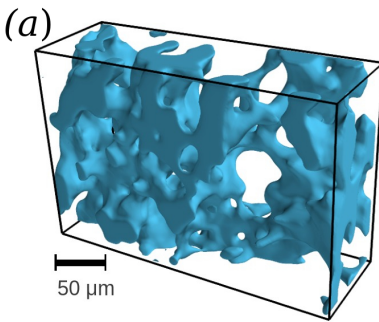

27 vol \% high viscosity phase

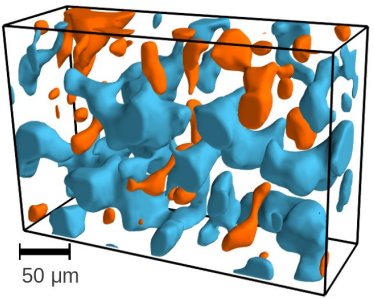

26 vol \% low viscosity phase

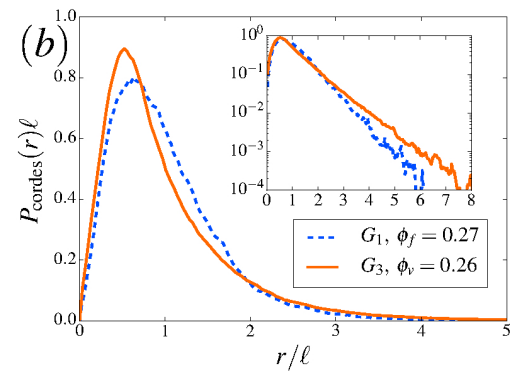

FIG. 3. (a) Morphology of the minority phase after $20 \mathrm{~min}$ at $1150^{\circ} \mathrm{C}$ for glasses $\mathrm{G}_{3}$ (Left) and $\mathrm{G}_{1}$ (Right). The largest connected domain is represented in blue; isolated domains in orange. When the minority phase is the fluid one $\left(G_{1}\right.$, $\Phi=0.27)$ a lot of isolated domains are present. This fragmentation process is absent when the minority phase is the viscous one $\left(\mathrm{G}_{3}, \Phi=0.72\right)$. (b) Chord distributions for the interconnected domains of (a), renormalized by the characteristic scale $\ell$. Semilog plot shown in inset.

$\mathrm{G}_{3}$. In Fig. 3(b), a quantitative view on this morphological contrast is given by the chord-length distribution [46] (the histogram of intercept lengths through the minority phase), with a more heterogeneous distribution and a longer tail for the viscous phase $\left(\mathrm{G}_{3}\right)$.

End pinching - While the simple scenario of endpinching presented above is appealing, the actual fragmentation mechanisms at play are likely to be more complex and interdependent due to hydrodynamic interactions. Yet, another phenomenon may result even more directly from end pinching: the refragmentation of already fragmented isolated domains. Although the geometry of these domains is not as well-controlled as that of the elongated drops used in the experimental fluid mechanics set-ups, we benefit here from a population of refragmenting drops significant enough for a statistical study. According to the end-pinching scenario [1, 2] an elongated droplet that relaxes can either relax to a sphere, or fragment in two or more droplets depending on the viscosity contrast and the aspect ratio.

The ratio of viscosity between a fluid droplet and the phase-separated mixture is low enough here to allow fragmentation. Still, a minimum extension is required for rupture to take place: no breaking event takes place below a threshold aspect ratio $L / R \lesssim 6$ where $L$ is the length of the elongated drop before relaxation and $R$ is a typical radius [1, 2]. The end-pinching scenario can be

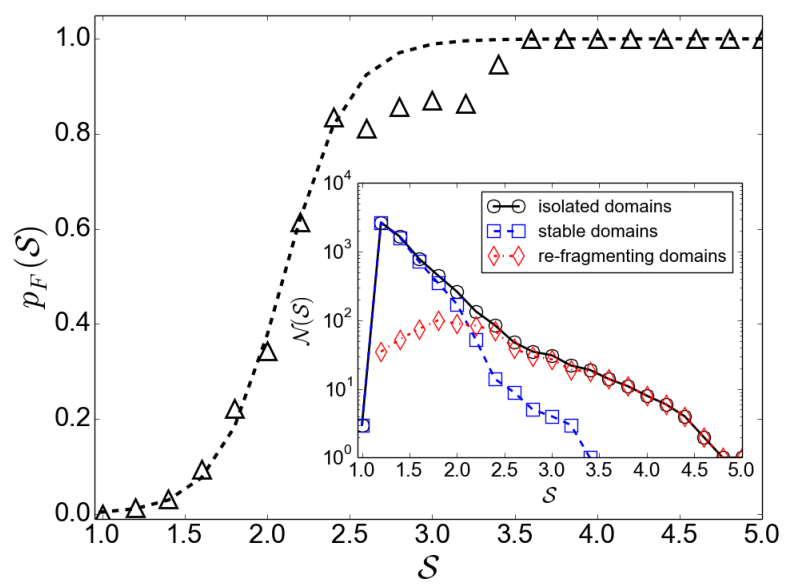

FIG. 4. Re-fragmenting probability of an isolated domain w.r.t. its sphericity index at first fragmentation (symbols). The dashed line shows a sigmoidal function $f(\mathscr{S})=1 /(1+$ $\left.e^{\left(\mathscr{S}_{-} \mathscr{S}_{c}\right) / w_{c}}\right)$ with $\mathscr{S}_{c}=2.1$ and $w_{c}=0.2$. Inset: Histograms of the sphericity index $\mathscr{S}$ at fragmentation of isolated domains (all, stable, refragmenting) of the three glasses $\mathrm{G}_{1}, \mathrm{G}_{2}$ and $\mathrm{G}_{3}$.

thus tested quantitatively via its dependence on the aspect ratio. In Fig. 4 we reported all fragmentation events from the interconnected cluster taking place along the coarsening of glasses $\mathrm{G}_{1}, \mathrm{G}_{2}$ and $\mathrm{G}_{3}$. In lieu of a ratio $L / R$ difficult to define on our domains we use a simple sphericity index $\mathscr{S}=S^{3 / 2} / 6 \sqrt{\pi} V$ where $S$ and $V$ are the surface and volume of the isolated domain just after it has detached from the infinite cluster. A spherical drop is such that $\mathscr{S}=1$ and an elongated domain is such that $\mathscr{S} \gg 1$. For a cylindrical drop of aspect ratio $L / R=6$, corresponding to the stability threshold of end-pinching, $\mathscr{S}_{c} \approx 4 / 3$. In the inset of Fig. 4 we show the sphericity histogram for the whole set of isolated domains at fragmentation as well as its restrictions to domains that do or do not refragment afterwards. The two populations are clearly distinguishable. In the main panel we show the cumulative probability of refragmenting versus sphericity. We get a sharp transition at $\mathscr{S}_{c} \approx 2.1 \pm 0.2$, a value a bit larger than for simple elongated droplets.

The scenario of end-pinching thus seems to reasonably account for the refragmentation of isolated domains. Despite the complexity of the material and the hightemperature conditions, these results support the idea that the viscous coarsening of silicate melts is fully accounted for by Newtonian fluid dynamics.

Conclusion - The dynamics of viscous coarsening appears to be dramatically modified by a strong viscosity contrast between the phases. A topological symmetry breaking is evidenced: elementary breaking events occur only in the most fluid phase and lead to fragmentation while resorption events occur only in the most viscous phase that remains inter-connected. The crucial importance of pinch-off mechanisms is illustrated by an end- 
pinching fragmentation taking place in the fluid phase.

Acnowledgements X-ray tomography experiments were performed on beamline ID19 (proposals HD-501, SC-3724, LTP MA-1876). We thank Elodie Boller, Davy Dalmas, Pierre Lhuissier and Luc Salvo for their experimental help, Laurent Limat for interesting discussions, and Alban Sauret for helpful comments and suggestions. Support of ANR project EDDAM ANR-11-BS09-027 is acknowledged.

[1] H. A. Stone, B. J. Bentley, and L. G. Leal, J. Fluid Mech. 173, 131 (1986).

[2] H. A. Stone and L. G. Leal, J. Fluid Mech. 198, 399 (1989)

[3] E. Villermaux, Annu. Rev. Fluid. Mech 39, 419 (2007).

[4] R. Seemann, M. Brinkmann, T. Pfohl, and S. Herminghaus, Rep. Prog. Phys. 75, 016601 (2012).

[5] F. Veron, Annu. Rev. Fluid Mech 47, 507 (2015).

[6] E. Villermaux, C.R. Mécanique 340, 555 (2012).

[7] H. M. Gonnermann, Annu. Rev. Earth Planet. Sci. 43, 431 (2015).

[8] D. Bouttes, E. Gouillart, E. Boller, D. Dalmas, and D. Vandembroucq, Phys. Rev. Lett. 112, 245701 (2014).

[9] D. Bouttes, O. Lambert, C. Claireaux, W. Woelfell, D. Dalmas, E. Gouillart, P. Lhuissier, L. Salvo, E. Boller, and D. Vandembroucq, Acta Mater. 92, 233 (2015).

[10] A. J. Bray, Phil. T. Roy. Soc. A 361, 781 (2003).

[11] A. J. Bray, Adv. Phys. 43 (1994).

[12] L. F. Cugliandolo, Physica A 389, 4360 (2010).

[13] A. Sicilia, J. Arenzon, A. J. Bray, and L. Cugliandolo, Phys. Rev. E 76, 1 (2007)

[14] E. D. Siggia, Physical Review A 20, 595 (1979).

[15] D. G. Aarts, R. P. Dullens, and H. N. W. Lekkerkerker, New J. Phys. 7, 40 (2005)

[16] L. K. Aagesen, A. E. Johnson, J. L. Fife, P. W. Voorhees, M. J. Miksis, S. O. Poulsen, E. M. Lauridsen, F. Marone, and M. Stampanoni, Nat. Phys. 6, 796 (2010).

[17] C. P. Royall, J. Eggers, A. Furukawa, and H. Tanaka, Phys. Rev. Lett. 114, 258302 (2015).

[18] D. Bouttes, Séparation de phase dans un verre borosilicate de baryum: du mûrissement à la fragmentation, Ph.D. thesis, Université Pierre et Marie Curie (2014).

[19] P. Levitz, Cem. Concr. Res. 37, 351 (2007).

[20] P. Levitz, V. Tariel, M. Stampanoni, and E. Gallucci, Eur. Phys. J. Appl. Phys. 60, 24202 (2012).
[21] M. Fialkowski, A. Aksimentiev, and R. Holyst, Phys. Rev. Lett. 86, 240 (2001).

[22] Y. Kwon, K. Thornton, and P. W. Voorhees, Europhys. Lett. 86, 46005 (2009).

[23] W. Nagel, J. Ohser, and K. Pischang, J. Microscopy 198, 54 (2000).

[24] C. Lang, J. Ohser, and R. Hilfer, J. Microscopy 203, 303 (2001).

[25] H.-J. Vogel, U. Weller, and S. Schlüter, Comput. Geosci. 36, $1236(2010)$

[26] S. Tomotika, Proc. Roy. Soc. A 10, 322 (1935); Proc. Roy. Soc. A 153, 302 (1936)

[27] J. R. Lister and H. A. Stone, Phys. Fluids 10, 2758 (1998).

[28] J.-W. Ha and L. G. Leal, Phys. Fluids 13, 1568 (2001).

[29] A. Y. Tong and Z. Wang, Phys. Fluids 19, 092101 (2007).

[30] S. Quan, D. P. Schmidt, J. Hua, and J. Lou, J. Fluid Mech. 640, 235 (2009).

[31] I. Cohen, M. P. Brenner, J. Eggers, and S. R. Nagel, Phys. Rev. Lett. 83, 1147 (1999).

[32] W. Zhang and J. Lister, Phys. Rev. Lett. 83, 1151 (1999)

[33] A. Sierou and J. R. Lister, J. Fluid Mech. 497, 381 (2003).

[34] J. Beaucourt, T. Biben, A. Leyrat, and C. Verdier, Phys. Rev. E 75, 1 (2007).

[35] M. R. Booty, D. T. Papageorgiou, M. Siegel, and Q. Wang, IMA J. Appl. Math. 78, 851 (2013).

[36] J. R. Castrejón-Pita, A. A. Castrejón-Pita, S. S. Thete, K. Sambath, I. M. Hutchings, J. Hinch, J. R. Lister, and O. A. Basaran, Proc. Nat. Acad. Sci. 112, 4582 (2015).

[37] J. Eggers and E. Villermaux, Rep. Prog. Phys. 71, 036601 (2008)

[38] E. Pairam and A. Fernández-Nieves, Phys. Rev. Lett. 102, 234501 (2009).

[39] H. Mehrabian and J. J. Feng, J. Fluid. Mech. 717, 281 (2013).

[40] K. Sarkar and W. R. Schowalter, J. Fluid Mech. 436, 207 (2001).

[41] N. E. Jackson and C. L. Tucker III, J. Rheol. 47, 659 (2003).

[42] S. Torquato, Random Heterogeneous Materials. Microstructure and Macroscopic Properties (Springer, New York, 2002).

[43] J. Eggers, Phys. Rev. Lett. 71, 3458 (1993).

[44] M. P. Brenner, J. R. Lister, and H. A. Stone, Phys. Fluids 8, 2827 (1996).

[45] J. Eggers and M. A. Fontelos, J. Fluid Mech. 530, 177 (2005).

[46] S. Torquato and B. Lu, Phys. Rev. E 47, 2950 (1993). 